\title{
Analysis of Prognostic Relevance based on Ambulatory Vital Signs Among Pregnancy with Pulmonary Hypertension in the ICU
}

\section{Renhuai Liu}

Fourth Military Medical University: Air Force Medical University

\section{Ziyu Zheng}

Changan University: Chang'an University

\section{Chen $\mathrm{He}$}

Fourth Military Medical University: Air Force Medical University

Chong Lei

Fourth Military Medical University: Air Force Medical University

\section{Yu Chen}

Fourth Military Medical University: Air Force Medical University

\section{Zongping Fang}

Fourth Military Medical University: Air Force Medical University

\section{Haopeng Zhang}

Fourth Military Medical University: Air Force Medical University

\section{Zhihong Lu}

Fourth Military Medical University: Air Force Medical University Huang Nie

Fourth Military Medical University: Air Force Medical University

\section{Dongyan Liang}

Fourth Military Medical University: Air Force Medical University Jun Tang

Fourth Military Medical University: Air Force Medical University

\section{Xiangdong $\mathrm{Ma}$}

Fourth Military Medical University: Air Force Medical University

\section{Hong Yang}

Fourth Military Medical University: Air Force Medical University

\section{Xijing Zhang}

Fourth Military Medical University: Air Force Medical University

\section{Binxiao Su ( $\nabla$ su_luccy@126.com )}

Fourth Military Medical University: Air Force Medical University https://orcid.org/0000-0002-0094-8569 
Research

Keywords: Ambulatory vital signs, pregnancy, Pulmonary hypertension, risk factors

Posted Date: September 13th, 2021

DOI: https://doi.org/10.21203/rs.3.rs-889429/v1

License: (c) (i) This work is licensed under a Creative Commons Attribution 4.0 International License. Read Full License 


\title{
Analysis of prognostic relevance based on ambulatory vital signs
}

\section{among pregnancy with pulmonary hypertension in the ICU}

Renhuai Liu ${ }^{1,3} \dagger$, Ziyu Zheng ${ }^{2} \dagger$, Chen $\mathrm{He}^{1,3}$, Chong Lei ${ }^{1,3}$, Yu Chen ${ }^{1,3}$, Zongping Fang ${ }^{1,3}$, Haopeng Zhang ${ }^{1}$, Zhihong $\mathrm{Lu}^{1}$, Huang $\mathrm{Nie}^{1}$, Dongyan Liang ${ }^{1,3}$, Jun Tang ${ }^{1,3}$, Xiangdong $\mathrm{Ma}^{4}$, Hong Yang ${ }^{4}$, Xijing Zhang ${ }^{1,3^{*}}$, Binxiao $\mathrm{Su}^{1,3^{*}}$

${ }^{1}$ Department of Anesthesiology and Perioperative Medicine, Xijing Hospital, Fourth Military Medical University, Xi'an, Shaanxi 710032, China

${ }^{2}$ Chang'an University, Xi'an, Shaanxi 710061 , China

${ }^{3}$ Department of Intensive Care Unit, Xijing Hospital, Fourth Military Medical University, Xi'an, Shaanxi 710032, China

${ }^{4}$ Department of Obstetrics and Gynaecology, Xijing Hospital, Fourth Military Medical University, Xi'an, Shaanxi 710032, China

\section{*Correspondence:}

Su Binxiao $3^{3 *}$, su_luccy@126.com; Xijing Zhang $3^{3 *}$, zhangxj918@163.com

$\dagger$ These authors contributed equally to this work.

\begin{abstract}
Purpose: The study seeks to utilise the extensively monitored data to explore the prognostic information in the continuous ambulatory vital signs of the pregnant women with pulmonary hypertension in the ICU, aiming to bring insights to physicians on evaluation and management of these specific patients.

Methods: This is a retrospective study of consecutive obstetric patients with PH admitted to ICU of the First Affiliated Hospital of Air Force Military Medical University of China, from January 2011 to May 2020. 92 cases are analysed via time-dependent Cox regression to take account of the dynamic features of vital signs.

Results : Seven out of 92 maternal deaths occurred, with most maternal deaths occurring within the first three days of admission to the ICU. The vital signs for the survived are more stable and normally ranged comparing to the death. Three vital signs are identified as risk factors in the maternal in-hospital mortality model: $\mathrm{SpO}_{2}(\mathrm{OR}, 0.93 ; 95 \% \mathrm{CI}, 0.89-0.98)$, Heart rate(OR,
\end{abstract}


$0.95 ; 95 \%$ CI , 0.92-0.99), Mean blood pressure(OR, 1.1; 95\%CI , 0.98-1). The model performance is justified by the ROC curve with AUC being 0.84. Further exploration showed that the total and the longest consecutive time ratios also affect the outcome.

Conclusions : Pregnancy women with $\mathrm{PH}$ who dead in hospital experienced long-term abnormal fluctuations in blood pressure, heart rate and blood oxygen during ICU stay. Both dynamic and time ratios reported impacts relating to $\mathrm{SpO}_{2}$, heart rate and blood pressure. In general $\mathrm{SpO}_{2}$ mitigate the hazard. Effects of the heart rate and mean blood pressure should be described combining the time ratios of hypotension and tachycardia.

Keywords: Ambulatory vital signs ; pregnancy ; Pulmonary hypertension; risk factors

\section{Introduction}

Pulmonary hypertension $(\mathrm{PH})$ is a progressive and devastating disease which often leads to right ventricular failure and death if untreated ${ }^{1}$. The great fluctuations in hemodynamics during pregnancy often aggravate such hemodynamic abnormalities for patients with $\mathrm{PH}$ conditions; particularly for those during deliveries or at early postpartum periods. These further increase the burden of the heart and further lead to maternal heart failures or even deaths ${ }^{2}$. Due to these reasons combined, the maternal mortality thus remains high (between $17 \%$ and $33 \%$ ) $^{3}$. Such mortality rate reaches especially high to around $50 \%$ for the pregnant women with Eisenmenger syndrome ${ }^{4}$; this severely affects the health and safety of pregnancy. As such, recent guidelines strongly recommend women with $\mathrm{PH}$ to avoid pregnancy for the health and safety reasons where possible . $^{5}$ However, some patients still either choose to become pregnant or continue with an unplanned pregnancy regardlessly. If such pregnancy is continued, it is crucial that such patients are referred to an expert $\mathrm{PH}$ centre for multidisciplinary management to receive close post-operative monitor in $\mathrm{ICU}^{6,7}$.Being one of the most resource-intensive units in hospital, continuous monitoring of patients provides access to abundant information contained in vital signs.

Considering that vital signs are the first step to prevention and management of critical illness ${ }^{8}$, they play crucial roles to recognisation of the patients at risks of deterioration. Hence, tracking dynamic vital signs to alert doctors to potential risk allows earlier actions to prevent potentially catastrophic maternal adverse outcomes. Previous studies have shown that the patients with $\mathrm{PH}$ are usually accompanied by hypoxia and hypotension ${ }^{9}$. Arrhythmias is also commonly seen in $\mathrm{PH}$ and associated with adverse outcome ${ }^{10}$. Unfortunately, quantitative validations of vital signs as risk factors towards the hazard of pregnant women with PH are still in scarcity with even fewer discussions over the effect size of them, not to mention the dynamically recorded vital signs. Therefore, this paper provides a different approach to investigation on the survival analysis of the pregnant women with $\mathrm{PH}$ via time-dependent Cox regression. A retrospective study on the admission to ICU of pregnancy with PH to further investigate the full potentials of ambulatory vital signs data is provided aiming to bring insights to physicians on evaluation and management of these specific patients.

\section{METHODS}

\section{Study subjects}

This is a retrospective study of consecutive obstetric patients with PH admitted to ICU of the First Affiliated Hospital of Air Force Military Medical University of China, from January 2011 to May 2020. All study subjects were either pregnant or within 42 days of postpartum, suffering from $\mathrm{PH}$ conditions. Admissions to ICU of 92 pregnant women with PH diagnosed were extracted from 
the electronic medical record system (EMRS) of the hospital in total. Further verification of these study subjects is done on supplement of the registeration books within the ICU department. The present study has been approved by the ethics committee ( KY20212019) . All information obtained were used only to describe the patient population and for data analysis. The study is registered in Chinese Clinical Trial Registry (ChiCTR2100046637).

\section{Data}

$\mathrm{PH}$ is defined as an increase in the mean pulmonary arterial pressure at rest (mPAP $\geq 25$ $\mathrm{mmHg}$ ) assessed by the right heart catheterization (RHC) ${ }^{11,12}$. Despite being an established gold standard tool of diagnostics for PAH, the RHC is an invasive procedure. Thus, for this study, the pulmonary arterial pressure is also monitored via echocardiography for all study subjects with $\mathrm{PH}$ on admission to ICU at the hospital. For consistency in the diagnostic criteria, all values are taken at the peak velocity tricuspid regurgitation when estimating the systolic pulmonary arterial pressure (sPAP). Although the 2015 ESC Guidelines for PH no longer recommend the SPAP as a diagnostic and stratification criterion, it is still widely used in clinical practice due to its intuitive and simple features. Hence in this study, all patients with right ventricular systolic pressure (RVSP) $>30 \mathrm{mmHg}$ at rest are noted as having $\mathrm{PH}^{13}$. Patients are further stratified into three groups based on RVSP: $30-50 \mathrm{mmHg}, 50-70 \mathrm{mmHg}$ and $>70 \mathrm{mmHg}{ }^{14,15}$. These measurement values are obtained using the echocardiography.

The following information were recorded in the registry: demographic characteristics, chief complaint; medical history, latest echocardiographic, childbearing history, mode of delivery, ante/postpartum admission and anesthetic mode. The information during the operation include the name of the operation, diagnosis upon ICU admission, Acute Physiology and Chronic Health Evaluation II(APACHE II) score. Vital signs are also continuously monitored in the ICU with the management in the ICU taken where appropriate. Other information include the length of stay in the ICU and hospital, conditions on discharge and so on.

\section{Statistical analysis}

All statistical analyses were performed using SPSS 26 (IBM Corporation, Armonk, New York, USA) and R software 4.0.5 (R Foundation for Statistical Computing, Vienna, Austria, 2016) . To handle the time-to-event data, this paper employs the technique of survival analysis where the response is taken to be the time between the entry of the study (admission to ICU) and the event (death or discharge from ICU). The Kaplan-Meier estimator is used to estimate and visualise survival probabilities as a function of survival time for exploratory analysis. Different from the previous studies, this paper utilises the dynamically monitored data on adaption of the Cox regression with time-dependent covariates to allow the inclusion of potential time-varying effects. Such model takes into account of the time-varying covariates $x(t)$ on which the hazard of death depends. By allowing time-varying effects, the model also automatically releases the assumption of proportionality and allows more flexible intake of factors. A $P$ value $<0.05$ was considered statistical significance.

\section{RESULTS}

\section{Baseline and clinic characteristics}

A total of 113 pregnant women diagnosed with PH admitted to ICU within the last decade in the First Affiliated Hospital of Air Force Military Medical University of China, 92 patients were finally analyzed. The mean maternal age of these cases is 27.05 , ranging between 17 and 42 . The 
mean gestational week of cases admitted to the ICU is $31.8 \pm 6.9$ where 63 of them $(68.5 \%)$ are nulliparous. The maternal in-hospital mortality is $7.6 \%$ where the most of the admissions were due to postpartum or miscarriage. There are 71 pregnancy cases that received cesarean section, 2 underwent vaginal delivery, 15 received Hysterotomy and 4 received therapeutic abortion . Out of them 67 were live birth and 48 were premature. The sPAP of the cases is $83.4 \pm 34.3$ where more than half of them (55.5\%) are classified to suffer from severe PH ( $\mathrm{PPAP}>70 \mathrm{mmHg})$. The APACHEII score of the cases on admission to the ICU is $9.1 \pm 5.2$. The average stay in ICU is around 2.9 days with an outlier of 14 days; $75 \%$ of the patients were discharged of ICU by 3 days in this study. Details of the basic and clinical characteristics of these cases are shown in Figure 1 and Table 1 respectively.

\section{Trends of vital signs}

The mean temporal trends across the survival and death groups over all time points within the study are shown in Figure 2. The vital signs for the survival group tend to be more stable and lie within the normal range in general comparing to the death. This is especially the case for $\mathrm{SpO}_{2}$ and HR where the death group presented lower $\mathrm{SpO}_{2}$ and higher HR values overall. Such observational differences between two trends as well as the fluctuations across times established the necessity to further look into the dynamic vital signs for pregnant women with $\mathrm{PH}$.

\section{Vital signs associated with death}

The dynamically monitored vital signs are analysed via the time-dependent Cox regression. Five relevant vital signs are selected using the backward selection procedure based on the AIC values. The most optimal model reached includes $\mathrm{SpO}_{2}, \mathrm{HR}$ and the MAP as predictors. Figure 3 shows that the hazard ratio for the selected vital signs monitored vary against time in this study. The coefficient estimates and hazard ratios can be found in Table 2 .

\section{Survival curve}

Under time-dependent Cox model, the survival curve adjusted for the three time-varying risk factors is shown in Figure 4. A clear drop is seen at around 72 hours with the rest of the times being rather flat. The survival probability remains above 0.9 at all times, agreeing to the initial descriptive data.

\section{Model diagnostics}

The corresponding the model outcome is further validated using ROC curve (Figure 5.). The AUC value reaches 0.84 which justifies a reasonable accuracy of model prediction.

\section{Additional exploration}

With the dynamically recorded data, it is possible that some values appear abnormal due to sudden incidence, or even due to mechanic failure of machines. Thus, this study further explores the hazard adjusted to the three selected vital signs with extension over the following two aspects: 1 . the percentage of the total time below a given threshold for each predictor of consideration (total time ratio) and 2. the percentage of the longest consecutive time below certain threshold for the relevant predictors (consecutive time ratio). As suggested by previous study, the clinical thresholds used for hypoxemia, tachycardia and hypotension are $\mathrm{SpO}_{2}<90 \%$, HR $>100 \mathrm{bmp}, \mathrm{MAP}<$ 
$65 \mathrm{mmHg}$ respectively.

For both the total and the consecutive time ratios, differences can be seen between the survived and death group (Figure 6.). This is especially the case for $\mathrm{SpO}_{2}<90 \%$ and $\mathrm{HR}>100 \mathrm{mbp}$. The total time ratio for $\mathrm{SpO}_{2}$ less than $90 \%$ shows higher median for the death group. The death group also experiences longer proportion of time with HR above 100bmp. Similarly for the consecutive time ratio, the death group suffers longer consecutive time of $\mathrm{SpO}_{2}<90 \%$ in general. The HR presents the similar pattern but slightly less obvious. The MAP time ratios, however, shows the least difference between the two groups in comparison to the other two.

The time-varying hazard ratio for each of total time ratios and the consecutive time ratios are shown in Figure 7. The effects are rather flat in comparison to the instantaneous measurements above (Figure 3). The estimates of hazard ratios are given in Table 3. Both hypoxemia and tachycardia contributes positively to the hazard; the consecutive time ratios clearly makes bigger impact in comparison. The forest plot for the total and consecutive time ratios for visualisation is given in Figure 8.

\section{DISCUSSION}

This paper successfully demonstrated an approach to investigation of survival behaviour of pregnant women with PH admitted to the ICU of the First Affiliated Hospital of Air Force Military Medical University of China with vital signs as risk factors identified. The study covers the period of January 2011 and May 2020 but is extensible to other times. A total of 92 cases with mortality rate being as high as $7.6 \%$ are discovered. Although the rate is quite a lot higher than 5/151(3.3\%) reported by the European Registry of pregnancy and Cardiac Disease (ROPAC) ${ }^{15}$. It is not unbelievable as the SPAP is suggest to associate with poor outcome ${ }^{16,17}$, this study contains a larger proportion of cases with severe $\mathrm{PH}$ (55.5\%).

Vital signs are shown to be significantly different statistically between the survived and dead cases. The averaged trend across individuals for all time points showed clear instability and wider range of fluctuation for the death group in general comparing to the survivals(Figure 2). Considering that the recording of vital signs in the ICU is a dynamic process and the hazard ratios do change over time (Figure 3), the use of static vital signs may not be capable to reveal the underlying effects involved. Thus given these observations, this article further established the relevant risk factors via time-dependent Cox regression. Such model not only respects the dynamic feature of vital signs but utilises all the information contained within the data. Such approach fully serves the demand of revealing the potentials of continuous vital signs data to assess the physiological state. At the same time, the time-dependent model diminishes the potential effects which might be brought into the final outcomes due to occasional mis-measurement.

Despite that in general, the vital signs averaged across individuals showed clear instability with wider ranges of fluctuation for the death group (Figure 2), the variables are not equally important when modelling the hazard. The final risk factors identified to contribute to the most optimal model fit when predicting the instantaneous $\mathrm{HR}$ are $\mathrm{SpO}_{2}$ (hazard ratio, $0.93 ; 95 \% \mathrm{CI}, 0.89$ - 
0.98), heart rate (hazard ratio, $0.95 ; 95 \% \mathrm{CI}, 0.92-0.99$ ) and the MAP (hazard ratio, 1.1; $95 \% \mathrm{CI}, 0.98-1.2)$. Both $\mathrm{SpO}_{2}$ and $\mathrm{HR}$ are statistically significant at $5 \%$ level and estimated to act adversely on hazard. For example, an increase in $\mathrm{SpO}_{2}$ by one unit tends to associate with hazard decrease by 0.02 . The MAP however shows different effect to the opposite direction. Despite being insignificant statistically the inclusion of it still improves the overall model behaviour. The interpretation of risk factor selections should be combined with the background information relating to clinical intervention in time series dynamics to further explain the clinical and physiological patterns found in the research ${ }^{18}$. Thus, in this study, one of the reasons that the MAP does not present as a significant predictor to hazard could be that most of the patients in the death group received the treatment of vasoactive drugs upon admissions to the ICU. This certainly brings manual intervention to the actual blood pressure. The lack of significant differences in respiratory rates between the two groups can also be explained by the use of ventilator. However, these should not mitigate the robustness and reasonableness of the study.

These estimates of the three vital signs agree with the observation of higher proportion of hypotension, tachycardia and hypoxia in the death group. More specifically, the dynamic $\mathrm{SpO}_{2}$ averaged over individuals (Figure 2) also showed that the death group fluctuates more in the hypoxic state, while the $\mathrm{SpO}_{2}$ of the survivals is mostly above $90 \%$; agreeing to the model results. These results fall in line with the previous finding where hypoxia is typically observed in the patients with $\mathrm{PH}^{19}$. The effect size of 0.93 means that the $\mathrm{SpO}_{2}$ affects the hazard ratio most in magnitude; this finding thus supports the conclusion of mixed venous oxygen saturation in $\mathrm{PH}$ may be a better prognosticator than cardiac index statistically ${ }^{20}$.

The HR affects the final outcome in the same direction to $\mathrm{SpO}_{2}$ but with slightly smaller effect size (0.95). Considering that the large fluctuation range of the HR observed in the death group, the conclusion of such cannot be made firmly. The hazard ratio of HR below 1 could be caused by the fact that patients at risk experienced increases in HR but still survived; especially when the previous recorded HR being extremely low. At the same time, the HR monitored drops dramatically at the end points towards deaths. Meanwhile, the most of patients had tachycardia associated to hypotension conditions suggested in ${ }^{21}$, the HR here may not indicate tachycardia but which patients are 'more tachycardia' in some sense. Therefore, how HR affects the hazard of death is a more complicated matter and it is potentially better described via the total or consecutive time ratios. As suggested in Figure 7, the death group does show a high percentage of time for tachycardia, agreeing to findings in ${ }^{21,22}$. The findings in total time and consecutive time ratios both suggested that when the HR remains above $100 \mathrm{bpm}$ for longer, the contribution to hazard of death increase. Similar to as suggested in the REVEAL 2.0 risk assessment, where the resting HR over $96 \mathrm{bpm}$ is one of the significant prognostic implications in PH patients ${ }^{22}$.

Knowing that the right heart failure being the principal determinant of death in pregnancy with $\mathrm{PH}$, hemodynamic changes during pregnancy in women with $\mathrm{PH}$ thus increase the burden of the heart and cause right heart failures. The model suggests that higher blood pressure tends to increase the hazard of death on the basis of MAP not less than $65 \mathrm{mmHg}$. This agrees with the exploratory analysis where the blood pressure trend in the death group is higher than the survival group overall. Therefore, the findings in this study does not violate the findings that systemic hypotension being 
one of the prognostic factors of poor outcomes in hospitalized patients ${ }^{23}$. Both the total and consecutive time ratios support the effects in the same manner. This also reminds us of the complexity of data analysis. The firm conclusions should be drawn based on observations and analysis from multiple dimensions.

This study successfully presented a way to assess the physiological state via continuously recorded vital signs in order to improve the clinical assessment of pregnant women with $\mathrm{PH}$. This should be further extended to more general cases in future studies considering that the monitoring of vital signs are comparably cheaper and easier. Especially when comparing to blood gas or laboratory tests, the vital signs can achieve real-time updates. It is already known that the vital signs of patients present useful information about physiological derangement and thus correlated to death ${ }^{24}$. At the same time, the vital signs are already used as the decisive information for clinicians at routine basis in $\mathrm{ICU}^{25}$. Future research taking account of dynamic trends of each vital signs should provide us with more generalised and valuable guidance not only in the area of pregnancy with PH but also other potential cases within ICU. The real-time evaluation of patients conditions is also achievable in the future with advanced monitoring equipment and better regularised recording systems.

\section{Study limitations}

This study has some limitations as being retrospective and deficiencies in data integrity. There might be a potential bias caused by the small sample size for cox regression modeling. This to certain extent limits the total number of study subjects in the paper considering $\mathrm{PH}$ being a rare disease in the pregnancy population. This however should not be a major limit to this study as the hospital from which the data is collected is the largest First-class Hospital at Grade 3 in the Northwest part of China, where the population accounts for most of the targeted patients within the region and thus is representative. The statistical model is rigorous and robust where the results need to be interpreted combining with other background information. All the data sources are authentic and reliable as the study was single-centric with original data recorded in a consistent way.

\section{Conclusion}

This study shows that pregnancy women with $\mathrm{PH}$ who dead in hospital experienced long-term abnormal fluctuations in $\mathrm{BP}, \mathrm{HR}$ and $\mathrm{SpO}_{2}$ during ICU stay. The ratio of total and longest consecutive duration of hypoxia, hypotension and tachycardia to length of ICU stay was higher in the death group than in the survival group. $\mathrm{BP}, \mathrm{HR}$, and $\mathrm{SpO}_{2}$ are predictors of death in a dynamic continuous vital signs multi-factor model. Continuous dynamic vital signs are time-sensitive and provide a predictive model with fewer risk factors.

Abbreviation: $\mathrm{AIC}=$ Akaike information criterion ; $\mathrm{AUC}=$ area under the receiver operating characteristic curve; APACHEII=acute physiology and chronic health evaluation II; $\mathrm{BP}=\mathrm{blood}$ pressure; $\mathrm{HR}=$ heart rate; $\mathrm{ICU}=$ intensive care unit; $\mathrm{MAP}=$ mean arterial pressure; NYHA: New York Heart Association ; $\mathrm{OR}=$ odds ratio ; $\mathrm{PAP}=$ pulmonary arterial pressure ; $\mathrm{PH}=$ pulmonary hypertension; $R O C=$ receiver operating characteristic curve; REVEAL $=$ Registry to Evaluate and Long-Term $\mathrm{PAH}$ disease management; $\mathrm{SD}=$ standard deviation; $\mathrm{SPAP}=$ pulmonary arterial systolic 
pressure;

\section{Acknowledgments}

Not applicable.

\section{Authors' contributions}

Renhuai Liu and Ziyu Zheng drafted the manuscript, had full access to all the data, and takes responsibility for the integrity of the data and the accuracy of the data analysis. Binxiao $\mathrm{Su}$ and Xijing Zhang contributed to study conception, design ,interpretation and critical revision. Chen $\mathrm{He}$, Jun Tang, Dongyan Liang contributed to data collection. Chong Lei,Yu Chen, Zongping Fang ,Haopeng Zhang, Zhihong Lu, Huang Nie, Xiangdong Ma, Hong Yang contributed to data interpretation and final approval of the manuscript.

Funding/Support : Dr Binxiao Su is supported by National Natural Science Foundation of China[grants H0910 81870961]

\section{Availability of data and materials}

The datasets used and/or analyzed during the current study are available from the corresponding author on reasonable request.

\section{Declarations}

\section{Ethics approval}

The study was approved by Ethic Committee of Xijing Hospital, Fourth Military Medical University (Approved NO. of ethic committee :KY20212019). As it was a retrospective analysis, consent to participate does not apply.

\section{Consent for publication}

Not applicable.

\section{Competing interests}

There is no conflict interest between authors

\section{References:}

1 Farber HW, Loscalzo J. Pulmonary arterial hypertension. N Engl J Med 2004; 351:1655-1665

2 Ramlakhan KP, Johnson MR, Roos-Hesselink JW. Pregnancy and cardiovascular disease. Nat Rev Cardiol 2020; 17:718-731

3 Duarte AG, Thomas S, Safdar Z, et al. Management of pulmonary arterial hypertension during pregnancy: a retrospective, multicenter experience. Chest 2013; 143:1330-1336

4 Bedard E, Dimopoulos K, Gatzoulis MA. Has there been any progress made on pregnancy outcomes among women with pulmonary arterial hypertension? Eur Heart J 2009; 30:256-265

5 Galiè N, Humbert M, Vachiery J, et al. 2015 ESC/ERS Guidelines for the diagnosis and treatment of pulmonary hypertension. Eur Respir J 2015; 46:903-975

6 SV K. Trends in pregnancy outcomes in patients with pulmonary hypertension: still a long way to go. Eur J Heart Fail 2016; 18:1129-1131

7 DG $\mathrm{K}, \mathrm{R} \mathrm{C}, \mathrm{V} \mathrm{W}$, et al. Improved survival in pregnancy and pulmonary hypertension using a multiprofessional approach. BJOG : an international journal of obstetrics and gynaecology 2010; 


\section{7:565-574}

8 Cummings MJ, Wamala JF, Bakamutumaho B, et al. Vital signs: the first step in prevention and management of critical illness in resource-limited settings. Intens Care Med 2016; 42:1519-1520

9 Olsson KM, Channick R. Pregnancy in pulmonary arterial hypertension. European Respiratory Review 2016; 25:431-437

$10 \mathrm{M} \mathrm{D}, \mathrm{H} \mathrm{N}, \mathrm{A} \mathrm{K}$, et al. Arrhythmias in adult patients with congenital heart disease and pulmonary arterial hypertension. Heart (British Cardiac Society) 2018; 104:1963-1969

11 MM H, HJ B, R C, et al. Definitions and diagnosis of pulmonary hypertension. J Am Coll Cardiol 2013; 62:D42-D50

12 G S, D M, DS C, et al. Haemodynamic definitions and updated clinical classification of pulmonary hypertension. The European respiratory journal 2019; 53

13 Strange G, Stewart S, Celermajer DS, et al. Threshold of Pulmonary Hypertension Associated With Increased Mortality. J Am Coll Cardiol 2019; 73:2660-2672

14 Martin SR, Edwards A. Pulmonary Hypertension and Pregnancy. Obstet Gynecol 2019; 134:974987

15 Sliwa K, van Hagen IM, Budts W, et al. Pulmonary hypertension and pregnancy outcomes: data from the Registry Of Pregnancy and Cardiac Disease (ROPAC) of the European Society of Cardiology. Eur J Heart Fail 2016; 18:1119-1128

16 MM H, T K, Z P, et al. Mortality in pulmonary arterial hypertension: prediction by the 2015 European pulmonary hypertension guidelines risk stratification model. The European respiratory journal 2017; 50

17 Frea S, Pidello S, Bovolo V, et al. Prognostic incremental role of right ventricular function in acute decompensation of advanced chronic heart failure. Eur J Heart Fail 2016; 18:564-572

18 Lehman LH, Adams RP, Mayaud L, et al. A Physiological Time Series Dynamics-Based Approach to Patient Monitoring and Outcome Prediction. Ieee J Biomed Health 2015; 19:1068-1076

19 MM H, MW P, H G, et al. Prognostic value of blood gas analyses in patients with idiopathic pulmonary arterial hypertension. The European respiratory journal 2007; 29:944-950

20 G K, A A, T N, et al. Mixed Venous Oxygen Saturation Is a Better Prognosticator Than Cardiac Index in Pulmonary Arterial Hypertension. Chest 2020; 158:2546-2555

21 JT M, A M, R L, et al. Arrhythmic Burden and Outcomes in Pulmonary Arterial Hypertension. Frontiers in medicine 2019; 6:169

22 RL B, M G, DP M, et al. The REVEAL Registry risk score calculator in patients newly diagnosed with pulmonary arterial hypertension. Chest 2012; 141:354-362

23 Inampudi C, Tedford RJ, Hemnes AR, et al. Treatment of right ventricular dysfunction and heart failure in pulmonary arterial hypertension. Cardiovascular Diagnosis and Therapy 2020;10:1659-1674

24 M B, S B, TV N, et al. Association between clinically abnormal observations and subsequent inhospital mortality: a prospective study. Resuscitation 2004; 62:137-141

25 BD W, SJ W, ER P, et al. Rapid-response systems as a patient safety strategy: a systematic review. Ann Intern Med 2013; 158:417-425

Table 1 Clinical characteristics of the study population

All patients
$(\mathrm{N}=92)$




\begin{tabular}{|c|c|}
\hline \multicolumn{2}{|c|}{ Baseline characteristics } \\
\hline Age, years(SD) & $27.05 \pm 5.7(17-42)$ \\
\hline Nulliparous & $63(68.5 \%)$ \\
\hline Gravidity & $2.1 \pm 1.2$ \\
\hline Gestational week & $31.8 \pm 6.9$ \\
\hline APCHE II score & $9.1 \pm 5.2$ \\
\hline sPAP & $83.4 \pm 34.3$ \\
\hline \multicolumn{2}{|l|}{ NYHA class } \\
\hline I & $53(57.6 \%)$ \\
\hline II & $11(12.0 \%)$ \\
\hline III & $16(17.4 \%)$ \\
\hline IV & $12(13.0 \%)$ \\
\hline \multicolumn{2}{|c|}{ Right ventricular systolic pressure $(\mathrm{mmHg})$} \\
\hline $30-50$ & $19(20.7 \%)$ \\
\hline $50-70$ & $22(23.9 \%)$ \\
\hline $70-90$ & $10(10.9 \%)$ \\
\hline$>90$ & $41(44.6 \%)$ \\
\hline \multicolumn{2}{|l|}{ Mode of delivery } \\
\hline Vaginal & $2(2.2 \%)$ \\
\hline Caesarean section & $71(77.2 \%)$ \\
\hline Hysterotomy & $15(16.3 \%)$ \\
\hline Abortion & $4(3.5 \%)$ \\
\hline \multicolumn{2}{|l|}{ Fetal outcome } \\
\hline Live birth & $67(72.8 \%)$ \\
\hline Premature & $48(52.2 \%)$ \\
\hline \multicolumn{2}{|c|}{$\begin{array}{lc}\text { Length of ICU stay, d } & 2.9 \pm 2.0(1-14) \\
\text { Maternal death, N }(\%) & 7(7.6 \%)\end{array}$} \\
\hline
\end{tabular}

NYHA: New York Heart Association

Table 2. Results of Multivariable cox regression analysis for the maternal in-hospital mortality.

\begin{tabular}{lllll}
\hline Predictors & $\beta$ & Hazard ratio & $\begin{array}{l}95 \% \text { Confidence } \\
\text { interval }\end{array}$ & $P$ Value \\
\hline $\mathrm{SpO}_{2}$ & -0.071 & 0.93 & $0.89-0.98$ & 0.004 \\
$\mathrm{HR}$ & -0.047 & 0.95 & $0.92-0.99$ & 0.008 \\
$\mathrm{MAP}$ & 0.071 & 1.1 & $0.98-1.2$ & 0.113 \\
\hline
\end{tabular}

Table 3: Hazard ratio of each thresholded predictors for total and consecutive time ratios

\begin{tabular}{|l|l|l|}
\hline Factor & Hazard ratio (Total) & Hazard ratio (Consecutive) \\
\hline hypoxemia & 1.004 & 215.02 \\
\hline tachycardia & 1.011 & $3.73 \times 10^{6}$ \\
\hline hypotension & 0.879 & 0.88 \\
\hline
\end{tabular}




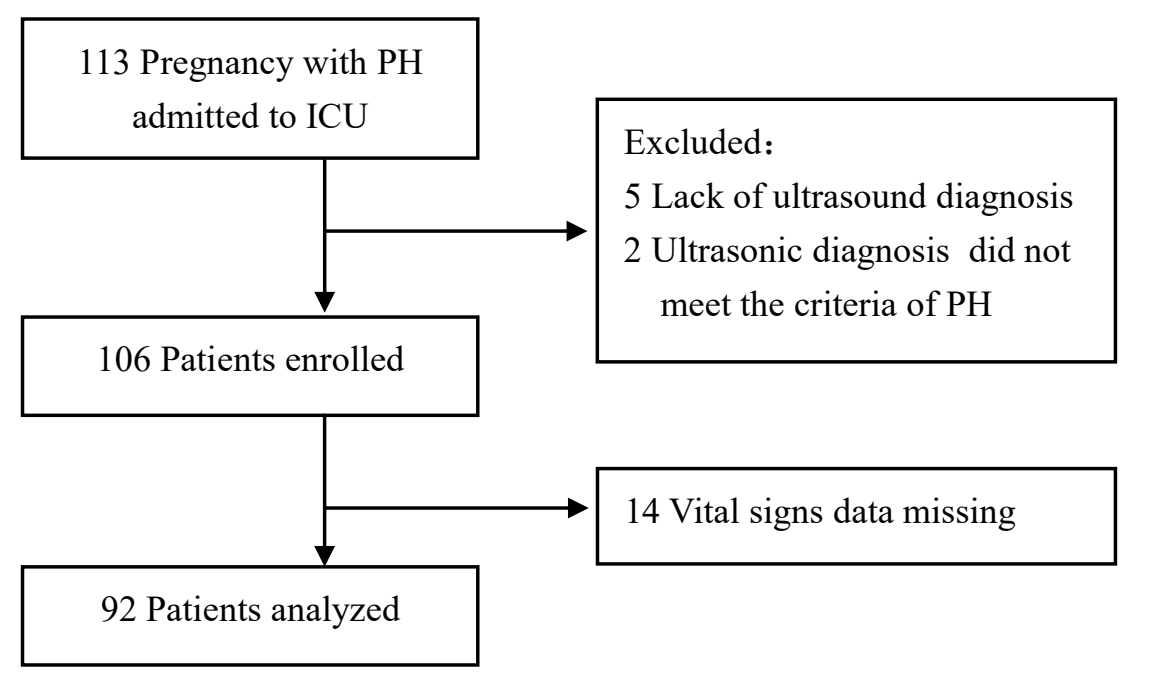

Figure 1. Flowchart of the study.

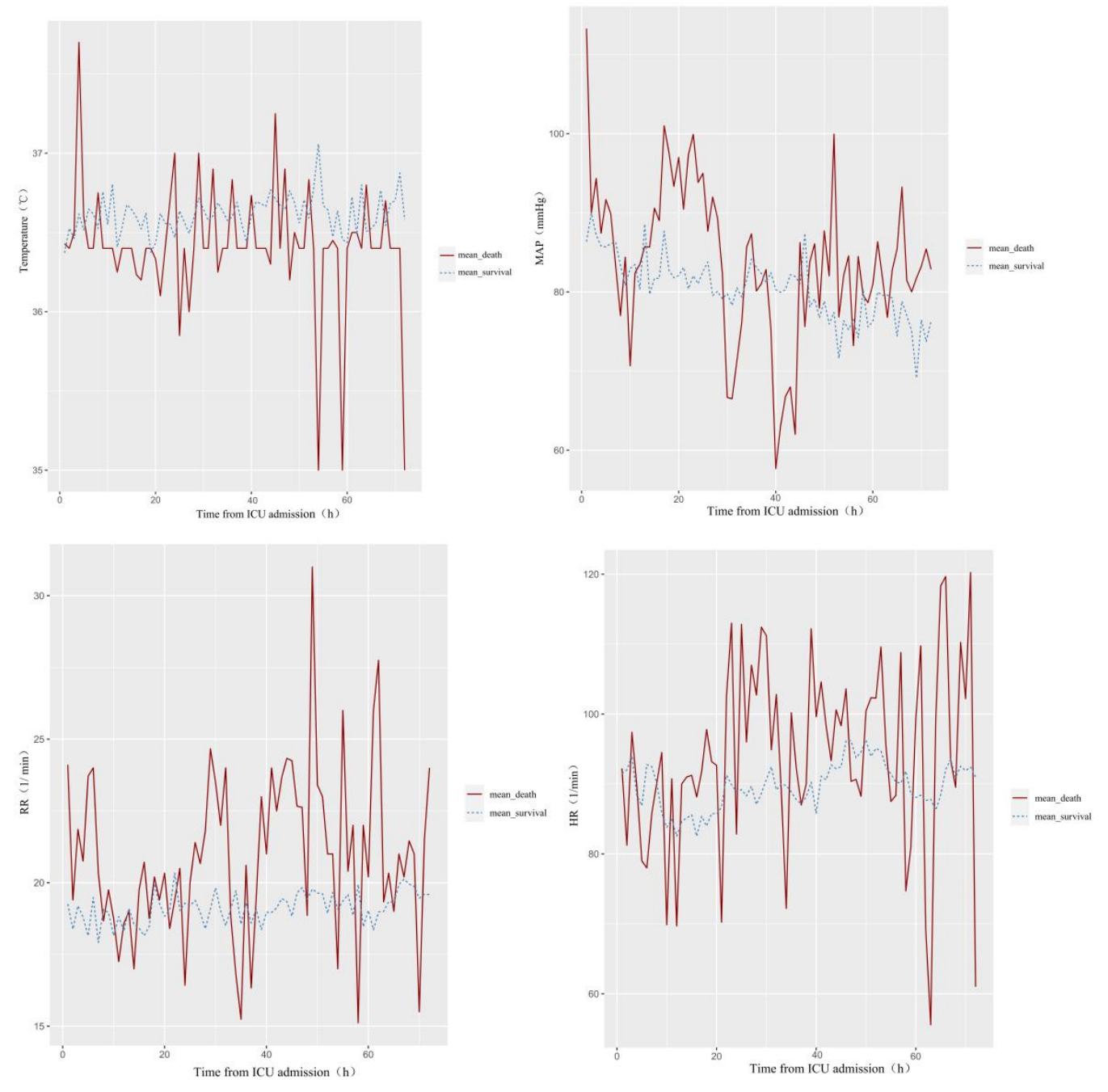




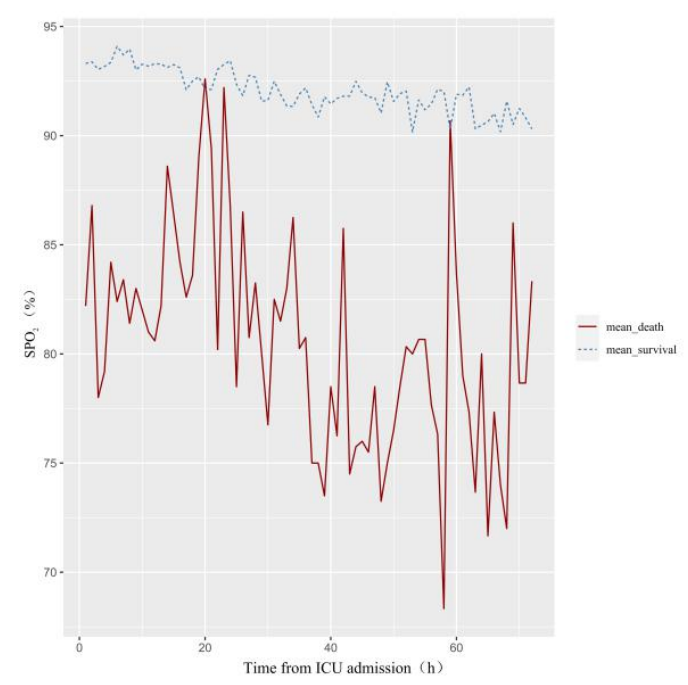

Figure 2. All patients were divided into the death group and the survival group according to the outcome of the ICU. The mean value of the vital signs at each time point of the two groups of patients was obtained after entering the ICU.
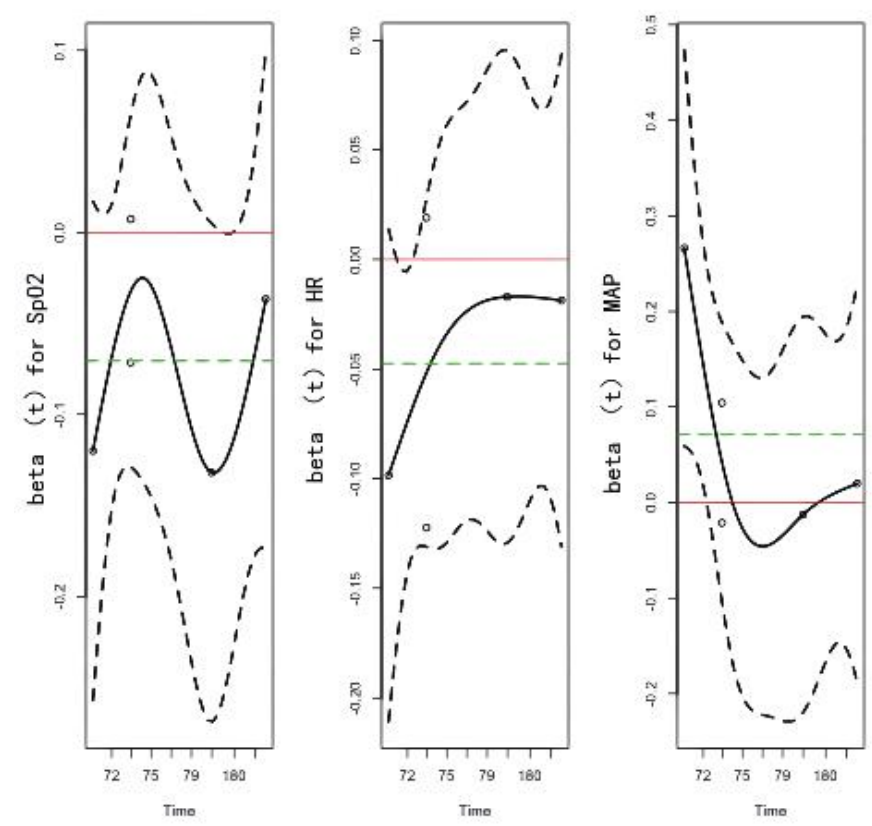

Figure3. Hazard ratio of $\mathrm{SpO}_{2}, \mathrm{HR}$ and MAP varying against time 

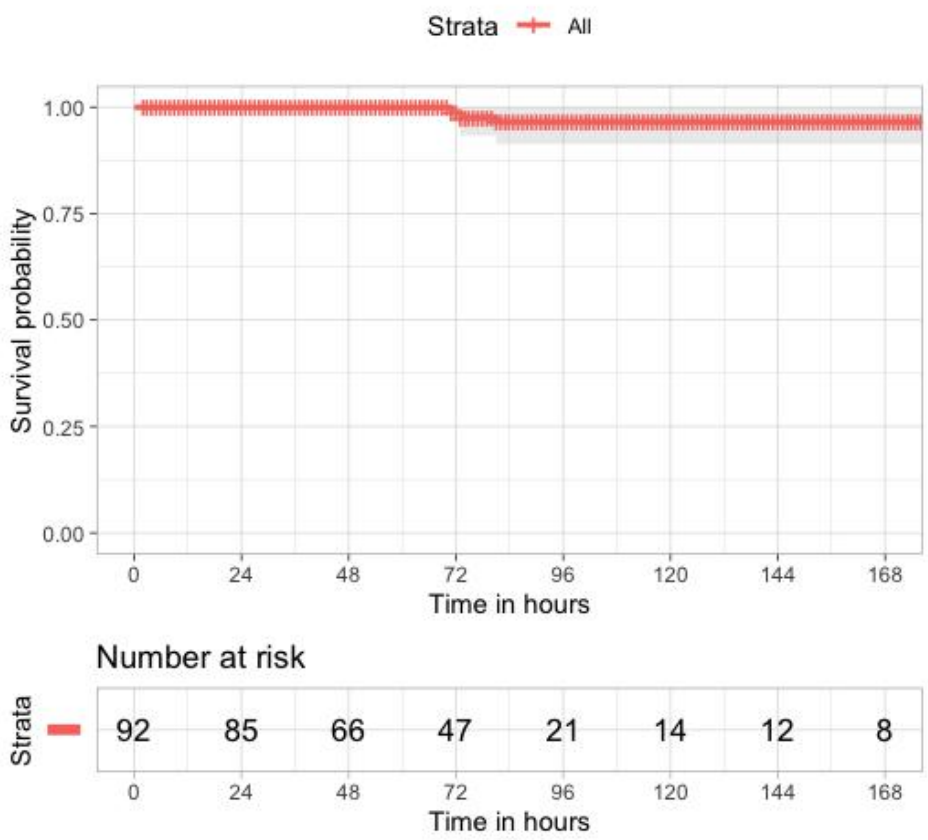

Figure 4. Survival curve adjusted for risk factors with risk table presented

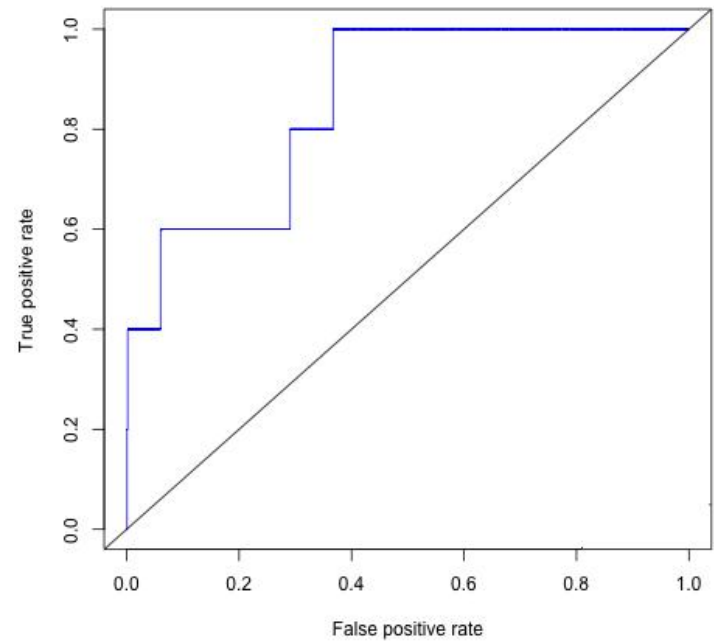

Figure 5. ROC: true positive vs false positive 

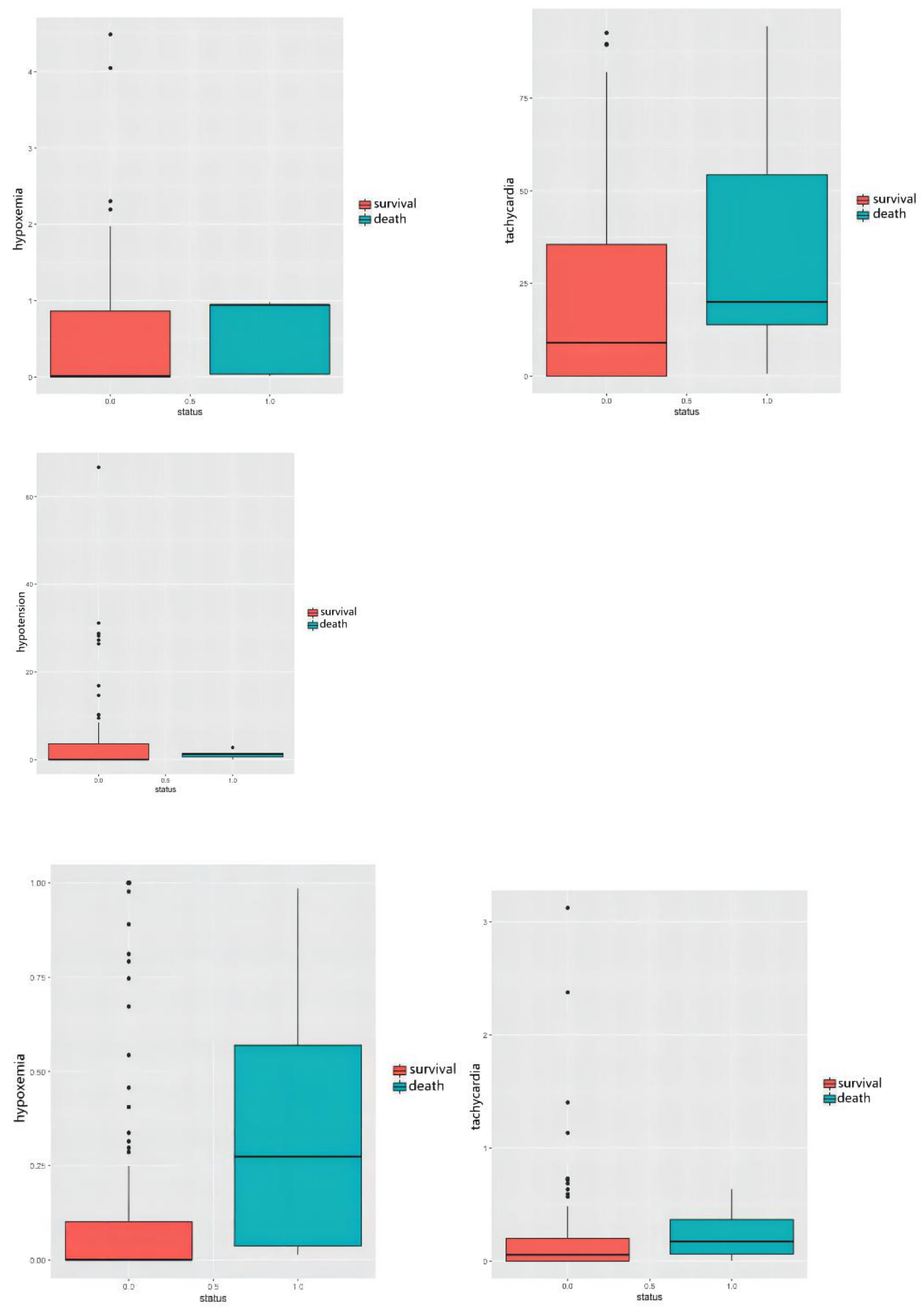


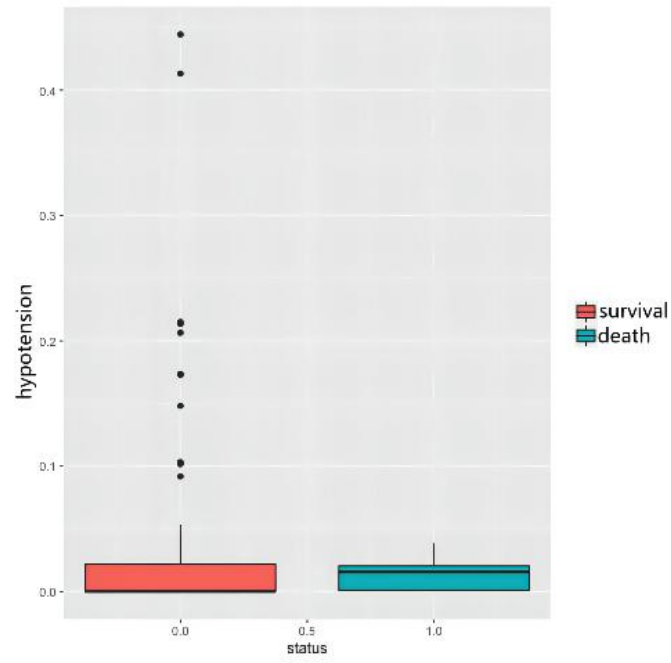

Figure 6. Boxplots for total and consecutive for hypoxemia, tachycardia and hypotension.
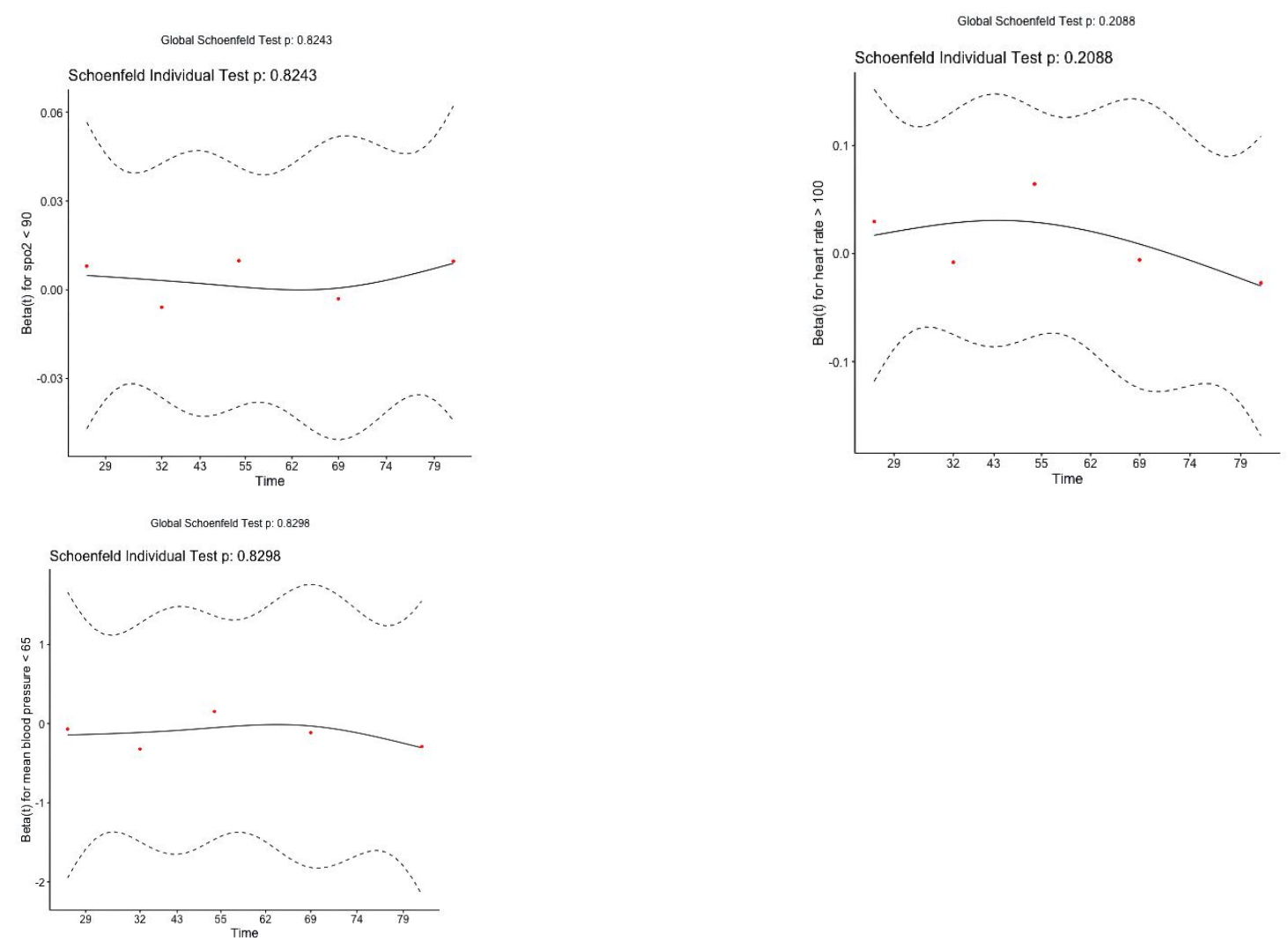
Glober schoenteld Test p: 05081
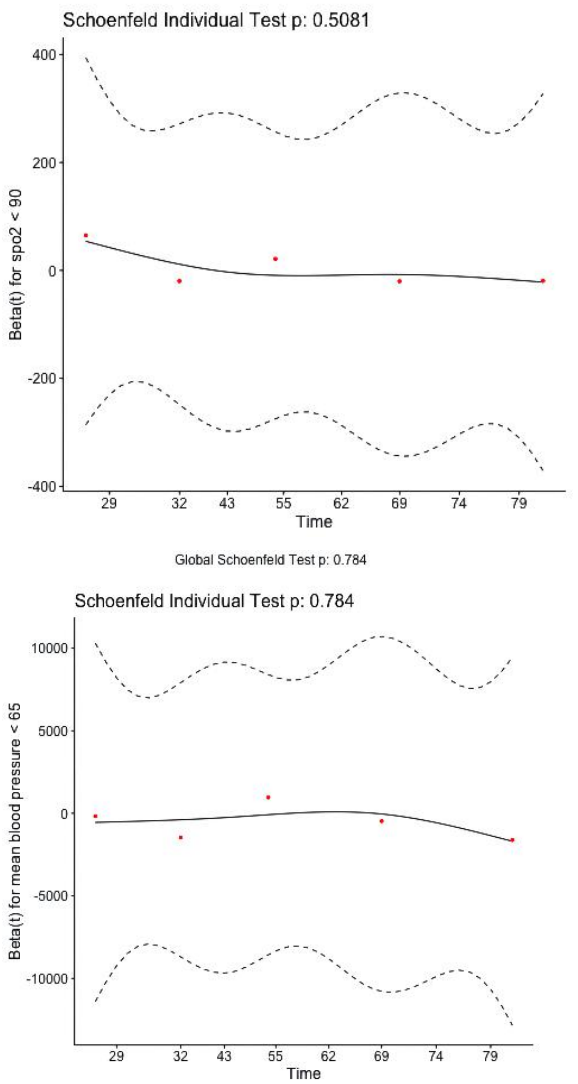

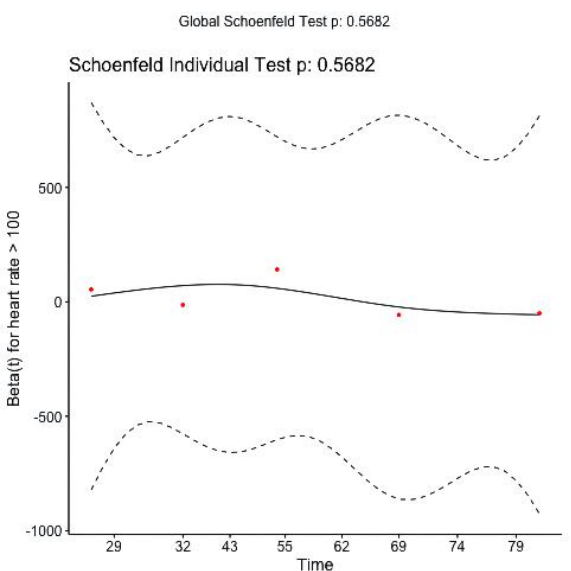

Figure 7. Hazard ratio variation with $95 \% \mathrm{CI}$ against times.

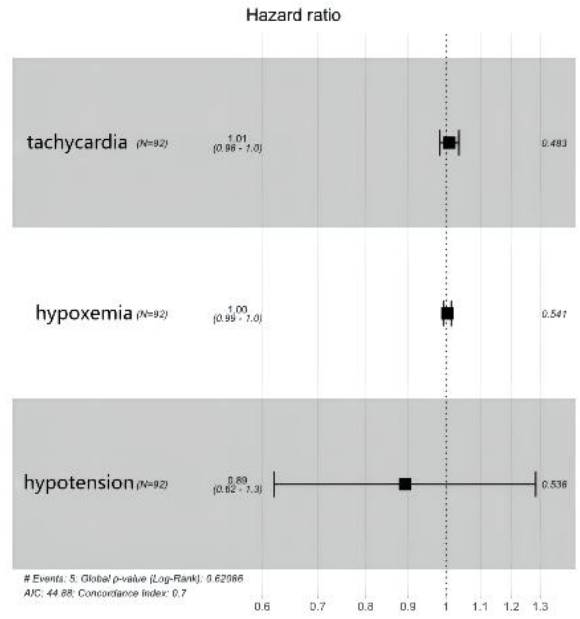

a

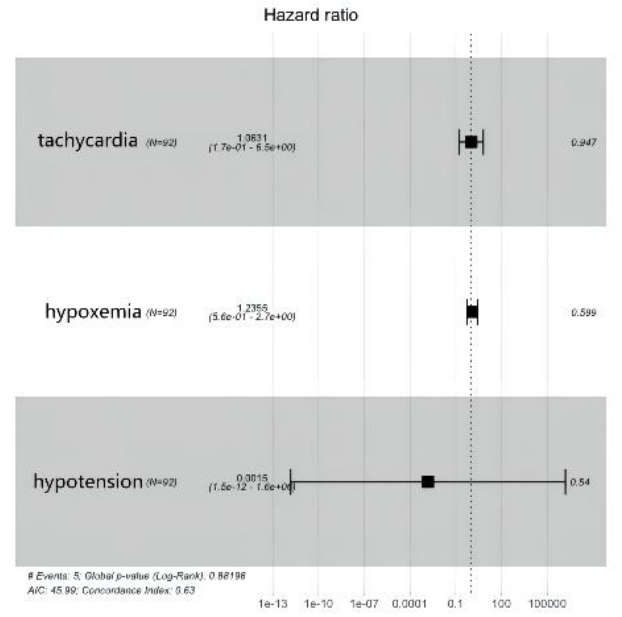

b

Figure 8: a. Hazard ratio with $95 \mathrm{CI}$ for percentage total b. Hazard ratio with $95 \mathrm{CI}$ for percentage consecutive 